\title{
Coronal mass ejection: key issues
}

\author{
Richard Harrison
}

Space Science and Technology Department, Rutherford Appleton Laboratory, Didcot, Oxfordshire OX11 0QX, United Kingdom, Email: richard.harrison@stfc.ac.uk

\begin{abstract}
Coronal Mass Ejections (CMEs) have been addressed by a particularly active research community in recent years. With the advent of the International Heliophysical Year and the new STEREO and Hinode missions, in addition to the on-going SOHO mission, CME research has taken centre stage in a renewed international effort. This review aims to touch on some key observational areas, and their interpretation. First, we consider coronal dimming, which has become synonymous with CME onsets, and stress that recent advances have heralded a move from a perceived association between the two phenomena to a firm, well-defined physical link. What this means for our understanding of CME modeling is discussed. Second, with the new STEREO observations, and noting the on-going SMEI observations, it is important to review the opening field of CME studies in the heliosphere. Finally, we discuss some specific points with regard to EIT-waves and the flare-CME relationship. In the opinion of the author, these issues cover key hot topics which need consideration for significant progress in the field.
\end{abstract}

Keywords. Sun: corona, Sun: coronal mass ejections, Interplanetary medium

\section{Introduction}

One of the solar physics 'grand questions' is, how are Coronal Mass Ejections (CMEs) initiated, how do they propagate and influence Solar System bodies? This is not a new question. There have been substantial efforts to address this and to obtain the relevant observations. It is clearly a multi-faceted question addressing the onset of CMEs, the physics of CME propagation into the heliosphere and impacts on bodies such as the Earth. Here we review key issues which are considered by the author to be critical for CME studies at this time. Thus, four quite specific questions are considered, namely:

1. What is the precise relationship between the CME onset and coronal dimming, and what can that tell us about the CME onset?

2. We now have observations of CMEs in the heliosphere; what are these telling us about CME propagation and impacts?

3. What is the relationship between CMEs and EIT waves, and does that relationship help us to understand the onset process?

4. Can we settle the issue of flare/CME asymmetry/symmetry?

\section{Coronal dimming - what does it really tell us?}

There is no strict definition of coronal dimming, even though it has been a well established topic of interest for some years. Perhaps the only definition that suits all of the reports to date would be: An extreme-UV (EUV) or X-ray intensity depletion of a large region of the corona. However, there are no generally accepted parameters for the degree of depletion, the size of the depletion area, or the EUV/X-ray wavelengths displaying depletion. This is not acceptable; rather loose or variable definitions will not help a proper 
interpretation of the physics behind these events. However, understanding the dimming may be of critical importance because they have been closely associated with CME onsets.

The dimming phenomenon is not a new discovery. Rust and Hildner (1976) reported a dimming event using Skylab observations. Dimming has been reported and analysed by many researchers using data from SOHO and Yohkoh, e.g. Sterling and Hudson (1997), Gopalswamy and Hanaoka (1998), Zarro et al. (1999), Harrison and Lyons (2000) and Harrison et al. (2003). Now, with the STEREO and Hinode spacecraft we are seeing the first reports of dimming using these spacecraft.

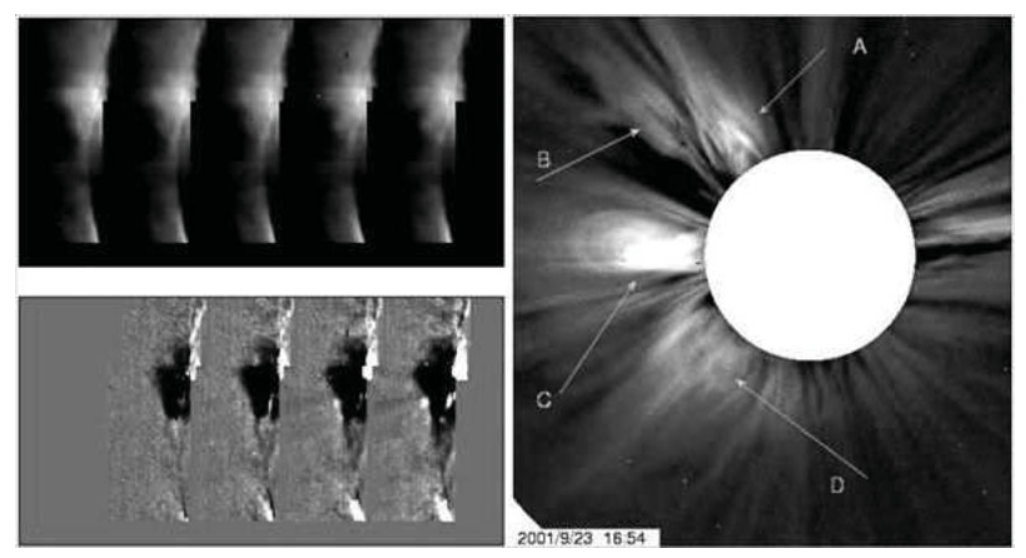

Figure 1. Dimming associated with a CME (Harrison, 2006). The SOHO/LASCO image (right) shows an east limb CME. A sequence of million $\mathrm{K} \mathrm{Mg}$ IX EUV images (top left) is shown with frames differenced from the first image (bottom left) to reveal dimming on the east limb.

Most papers referring to dimming rely on phenomenological associations. Timing and co-location or alignment with CME activity is discussed but little is said about the physical parameters of the dimming region. So, what is the physical relationship between the dimming and an associated CME? Does the dimming reveal the site of 'lost' mass seen later as (part of) the CME? If so, a determination of the parameters of the dimming plasma, and its history, is key to understanding the CME onset, but this stresses that we need plasma parameters and that requires spectroscopic observations. Thus, we stress the value of the studies utilising spectrometers aboard SOHO and Hinode.

There are studies which clearly indicate, from spectral analysis, that the dimming is due to density depletion (Harrison and Lyons 2000; Harrison et al. 2003). These studies also show that the mass loss, again calculated through spectroscopic analysis, is consistent with the associated CME mass, suggesting that we are seeing some or all of the plasma from the dimming as the ascending CME. Similar mass-loss calculations can be made using wide-band EUV/X-ray imager data but only with significant assumptions about the temperature of the plasma. However, it is the author's opinion that the earlier spectroscopic mass calculations do require further confirmation with additional studies using SOHO and Hinode.

Spectroscopic and imaging observations of dimming confirm that the relative timing between the dimming and CME, as well as co-location, show that the dimming events are temporally and spatially associated with the CME activity. These findings allow us to consider physical processes at work but there are two important aspects that we have been missing. These have been addressed recently by Bewsher et al. (2008). They produced the first statistical and probability spectroscopic study of the dimming phenomenon, 
utilising data from almost 200 observational runs using SOHO and utilising an automated dimming identification scheme. Applying an automated scheme means that Bewsher et al. (2008) have applied criteria for defining a dimming event. These criteria were selected based on experience from previous observations, and are an attempt to put some constraints on what we believe a dimming event to be. They identified dimming events by the 'depth' of the intensity drop and by the physical size in the image - specifically the minimum depth was twice the statistical error of the intensity measurement and the minimum area was $1.44 \operatorname{arcsec}^{2}$ (scale about 5 degrees on the Sun).

Bewsher et al.'s first goal was to put the dimming-CME association on a firm footing. There are many reports on individual events, or few events, but no statistical studies to establish the degree of the association. What Bewsher et al. (2008) found was that up to $84 \%$ of the CMEs in the observation periods could be projected back to dimming regions, and that is the first robust confirmation of the association between CMEs and dimming; these phenomena are clearly related.

The second major point made by Bewsher et al. (2008) utilised the spectral capabilities of their dataset. They identified 155 and 146 dimming events in the spectral lines of Mg IX at $368 \AA$ and Fe XVI at $360 \AA$, respectively. The abundances of $\mathrm{Mg}$ IX and Fe XVI peak at 1 and 2 million $\mathrm{K}$, respectively. In only 96 cases did the code identify the dimming in both lines. This means that for 59 of the million $\mathrm{K}$ dimmings, no dimming was seen at 2 million $\mathrm{K}$, and for 50 of the 2 million $\mathrm{K}$ dimmings, no dimming was identified at 1 million K. This confirms a point made by Harrison et al. (2003); there are significant variations in the degree of dimming between temperatures. Given adequate spectral (temperature) coverage, we can in principle identify all dimming events, but imaging from one wavelength will miss dimming events. There are clearly limitations on dimming identification from imagers alone unless multiple bands are used.

Finally, if dimming is revealing mass loss, can we observe the evacuation process? Harra et al. (2007) reported $40 \mathrm{~km} / \mathrm{s}$ blue-shifted outflows from a dimming region using EIS/Hinode data. This is consistent with Harra and Sterling's (2001) claims to have detected outflows from such a region using CDS/SOHO. Also consistent with this, Harrison and Bewsher (2007) reported CDS/SOHO limb observations of pre-flare diffuse loops ascending from a region as it decayed in intensity, i.e. became a dimming event (see Figure 2).

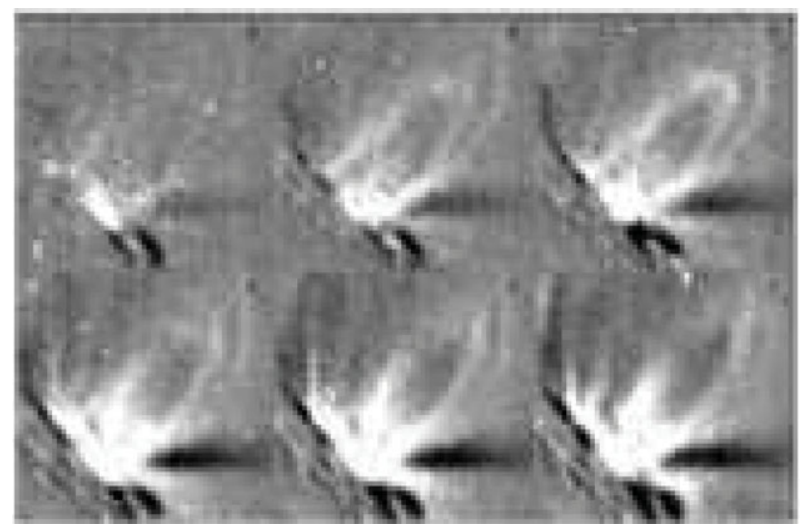

Figure 2. Pre-flare/pre-CME ascending loops detected using SOHO/CDS on 25 July 1999. These rising loops appear to reveal the evacuation of the corona, i.e. the dimming process. These are $4 \times 4$ arcmin images in the million K Mg IX line. (Harrison and Bewsher, 2007). 
This review of aspects of the dimming observations stresses some key points:

1. The first statistical analysis of a large spectroscopic dataset of dimming has put the CME-onset/dimming association on a firm footing; models must be consistent with this.

2. The same study shows that the degree of dimming varies with temperature between events. This must be taken into account, especially with the use of imager data.

3. Some studies appear to reveal the evacuation process itself as clear mass-outflows or even ascending loops. A key observation for the future is to extend that work.

4. Spectral studies confirm that dimming is due to mass-loss. The consistency between the lost mass and the associated CME mass suggests that the dimming region is the source of at least part of the CME. Again, we do need more observational confirmation of this.

5 . There are many consistent observations of relative timing and location that stress the association between the dimming and CME onsets.

\section{CMEs in the heliosphere - the potential of new observations}

Until recently, observations of CMEs in the heliosphere, including near-Earth space, have been effectively limited to single-point in-situ measurements. With the launch of the SMEI instrument aboard Coriolis, in 2003 (Eyles et al. 2003), and the Heliospheric Imagers (HIs) (Harrison et al. 2008), launched in 2006 aboard the two STEREO spacecraft, we now have wide-angle imaging of the heliosphere with the ability to detect CMEs out to beyond $1 \mathrm{AU}$. In particular, the HI instruments provide views from out of the Sun-Earth line, allowing studies of CMEs entering near-Earth space.

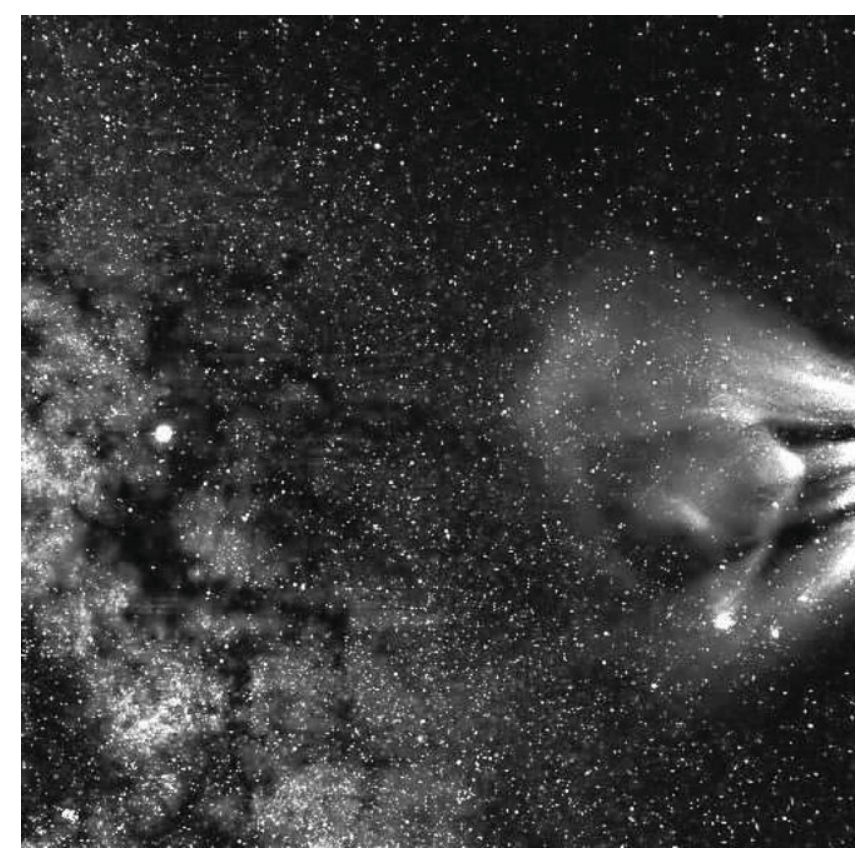

Figure 3. A CME in the heliosphere detected using HI-1 aboard STEREO A on 5 November 2007 (Harrison et al. 2009a). The image is 20 degrees across. The horizontal centre-line defines the ecliptic plane. The Sun is 4 degrees off the right hand side. The Milky Way is visible on the left of the frame, with Jupiter at centre-left. Stars down to 12th magnitude can be seen. A CME is clearly seen on the right hand side of the frame. 
Figure 3 (from Harrison et al. 2009a) shows the quality of the HI instruments; the baffling systems are such that scattered light levels are reduced to $10^{-13}$ of the solar brightness at worst (Eyles et al. 2008). This, combined with the instrument sensitivity allows the imaging of stars down to 12th magnitude. The image shows a range of Solar System and stellar bodies, but the feature of primary interest is the CME on the right hand side of the frame.

The HIs allow imaging from elongations of a few degrees from Sun-centre out to almost 90 degrees. They provide us with the first real chance to study CMEs as they pass through the inner heliosphere and near-Earth space. A number of CMEs are discussed by Harrison et al. $(2008,2009 \mathrm{a})$ and we show a few examples in Figure 4. The HI instruments on each spacecraft are identical. Each consists of two telescope systems, one viewing a $20 \times 20$ degree field centred on the ecliptic plane from 4 to 24 degrees elongation, and the other occupying a $70 \times 70$ degree field, also centred on the ecliptic plane, in this case from 19 to 89 degrees. The instrument concept, fields of view and operation are described by Harrison et al. (2008) and Eyles et al (2009).

Harrison et al. (2009b) have reviewed the CME/ICME relationship using the new HI data and building on early HI results reported by Harrison et al. (2008). In a sense, a CME is considered to be a near-Sun eruption imaged using a coronagraph and an Interplanetary CME (ICME) is a mass ejection in the heliosphere, traditionally only viewed using in-situ measurements. Crooker and Horbury (2006) discussed the connectivity of ICMEs to the Sun, based on interpretations from the in-situ data. It is widely accepted that counter-streaming particle beams in ICMEs indicate that both ends of the ICME are indeed connected to the Sun. On the other hand, uni-directional beams may signal connection at only one end. Logically, then, the lack of beams would appear to signal disconnection at both ends. In this case the ICME has become an isolated plasmoid. The in-situ observations suggest that most ICMEs are connected at both footpoints for a considerable time after the eruption. There is evidence for closed ICMEs even out to Jupiter-like distances. This interpretation is indeed supported by the new HI data which demonstrate the long-duration connectivity of individual CMEs in the heliosphere, at least to Earth-like distances, and stress the fact that there is no evidence for magnetic pinching off of CMEs (Figure 5, left hand panel).

However, McComas (1995) has argued that the heliospheric magnetic flux does not continually build up. Flux must be shed through reconnection somehow. How is this consistent with the lack of observation of closing down magnetic systems behind ascending CMEs? The answer may be in the form of an interchange reconnection process, which has been suggested by Gosling et al. (1995) (Figure 5). The idea is that the ascending CME can travel a considerable distance still connected to the Sun, and that days or even weeks after the onset, the legs of the CME, still rooted in the Sun, will interact with adjacent open field lines at low altitude in the corona; reconnection results in the formation of low-lying loops and an outward ascending kink-shaped structure ascends into the heliosphere from the site of one of the original CME footpoints. In Figure 5, this is contrasted with the traditional approach where the ascending CME is magnetically pinched off, resulting in an ascending plasmoid and loops closing down underneath. The interchange mechanism has the attractive feature that the site of the greatest field density, magnetic complexity and field-line motion is the site of reconnection. Harrison et al. (2009) argue that although this results in the outward propagation of a kinked field-line configuration, what we might expect to observe with coronagraphs or using HI, would be narrow $\mathrm{V}$-shaped, ascending features or trains of ascending blobs associated with reconnection in the boundaries between closed and open fields; the CME front would be 

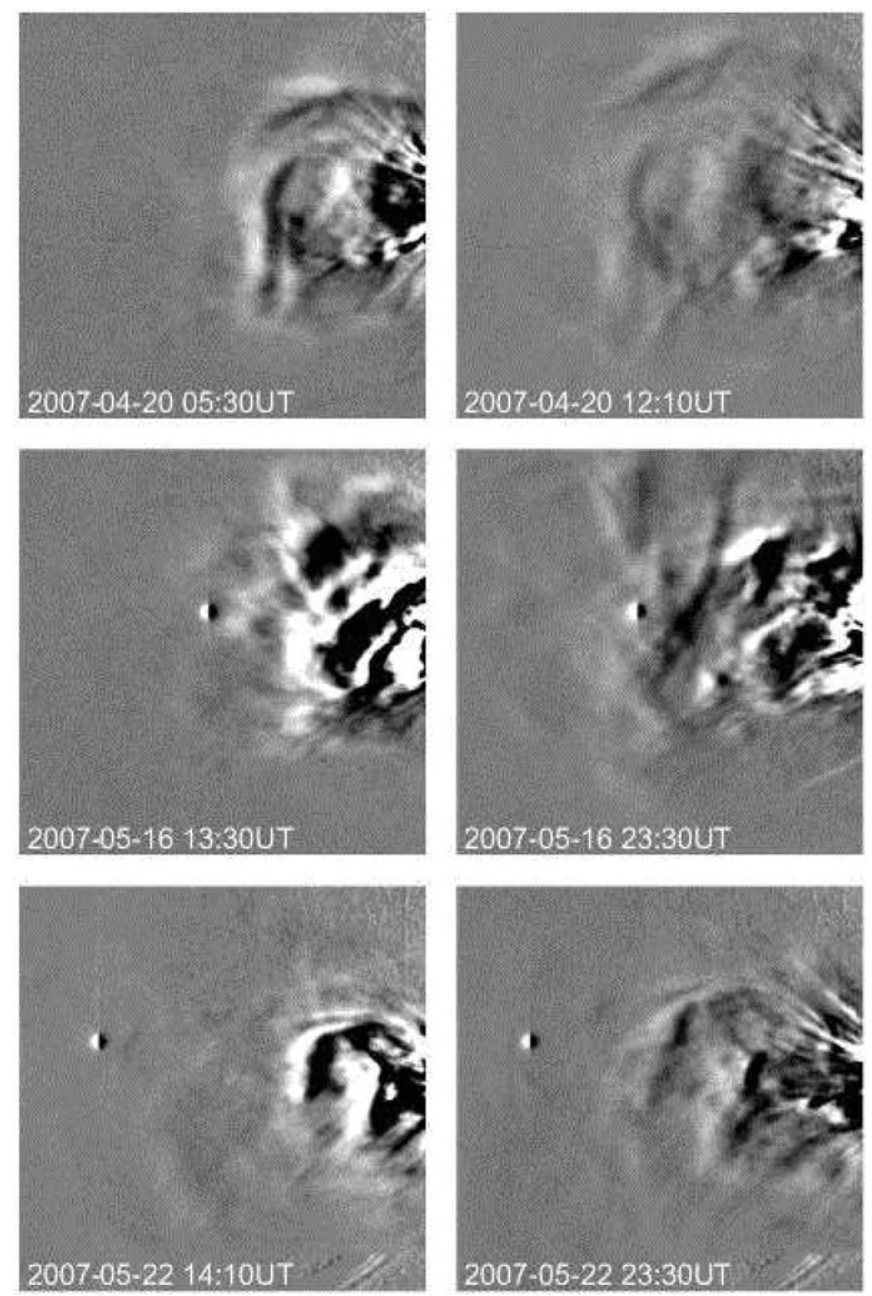

Figure 4. Three CMEs imaged using HI-1, on 20 April, 16 May and 22 May 2007. For each, two frames show their passage through the first 20 degrees elongation from the Sun. These images show that the same basic structure persists.

long gone. Evidence for such phenomena is being sought at this time and could prove to be crucial in understanding the CME process.

Another valuable analysis of the HI data is providing a global view of CME activity in the inner heliosphere. Davies et al. (2009) have extended the coronagraph time-altitude plots due to Sheeley et al. (1999) to produce a time-elongation display which shows the nature of CME activity from 4 to almost 90 degrees elongation. Figure 6, from Davies et al. (2009), shows such a plot which is produced from stacking intensity scans along the ecliptic plane across the HI-1 and HI-2 data. Outward propagating events are revealed by sloping lines whose gradients and shapes are a function of the outward speed and the location of the event with respect to the plane of the sky (Rouillard et al. 2008a). Such a plot extends this technique by an order of magnitude from the Sun, over previous studies. The method reveals a plethora of ascending structures in the lowest portions of the image (below 10 degrees elongation - i.e. in the region occupied by the coronagraph fields). From a few tens of degrees we detect far fewer events. Outward propagating features 


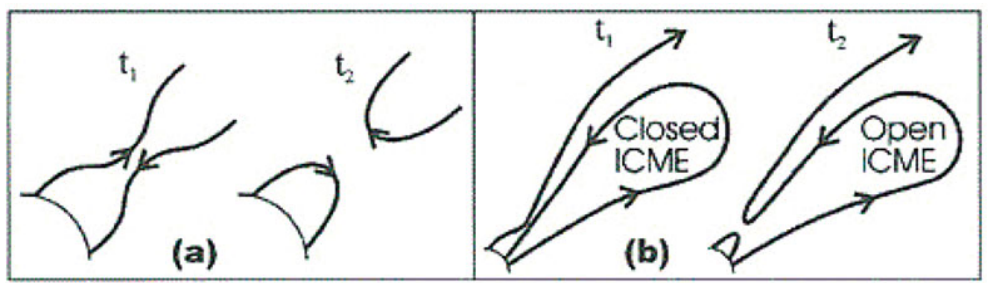

Figure 5. (a) The traditional view of an ascending CME pinching-off, and (b) the interchange reconnection process (from Crooker and Horbury 2006).

may be dissipating or decreasing in intensity as they expand outwards. Observationally, events are best viewed if they are located on the so-called Thomson sphere, defined as the circle or sphere with the Sun-Spacecraft line as the centre-line. This sphere defines the points of 90 degree scatter of the photospheric light off free electrons in the heliosphere to the 'observer' at the STEREO spacecraft (Vourlidas and Howard, 2006). If the forest of ascending structures at the lowest levels of Figure 6 are typical of all longitudes then the effect of the Thomson sphere on interpreting this will not be of great significance.

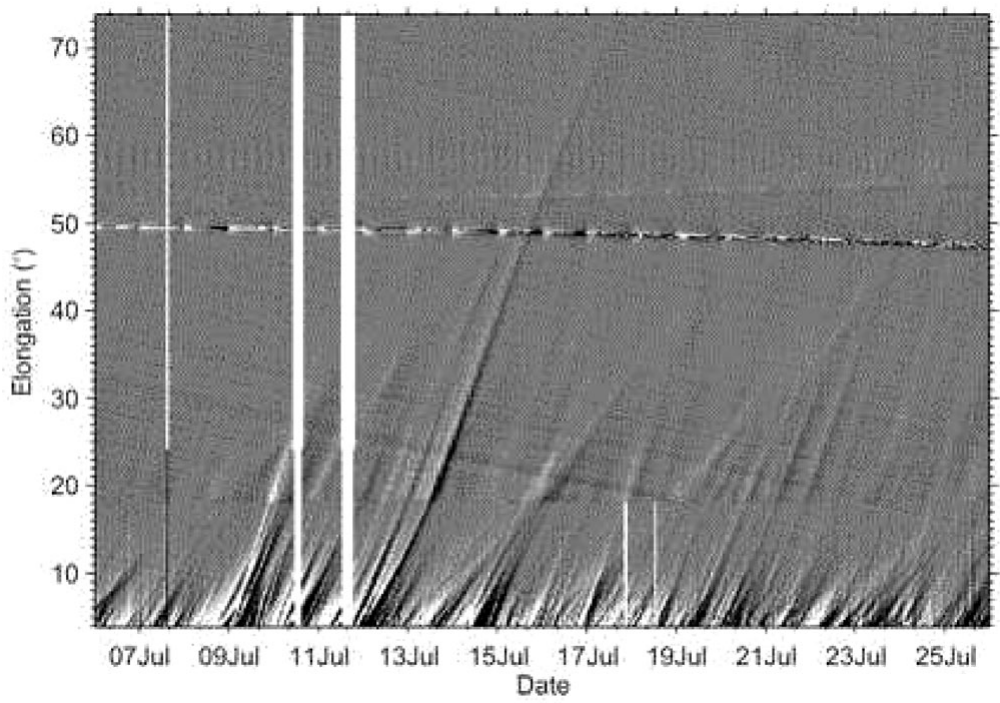

Figure 6. A time-elongation plot utilising the HI-1 and HI-2 data from STEREO A for July 2007 (from Davies et al. 2009)

Above 50 degrees elongation we detect just a few ascending structures. Just one event crosses the entire image to the outer edge of the field. The slope and shape of the profile show that this CME ascended at $320 \mathrm{~km} / \mathrm{s}, 48$ degrees east of the Spacecraft-Sun line (Davies et al. 2009), which is consistent with an association with an active region at E29 (longitude from Earth).

Plots such as these do show a synoptic and global view of ejecta in the inner heliosphere, demonstrating a simplification of the ascending structure with elongation. This also stresses the value of in-situ measurement near to the Sun to sample pristine solar wind, and this is one of the prime goals of the Solar Orbiter mission. 
Whilst considering CMEs in the heliosphere, we must consider their impact on Solar System bodies. A graphic illustration of this has been presented by Vourlidas et al. (2007). They show the impact of a CME on comet Encke, which resulted in a complete disconnection of the comet's ion tail, presumably due to the CME magnetic fields interacting with the cometary plasma. This is a demonstration on a small scale of events which could occur with other bodies, including planets.

This short discussion demonstrates the potential for this observational approach. We note that there are on-going studies of planetary impacts of CMEs, as well as other cometary studies and imaging of Co-rotating Interaction Regions (see Rouillard et al. 2008a,b; Sheeley 2008a,b).

In conclusion, wide-angle heliospheric imaging is providing us with many new lines of study. Of particular interest is the propagation of CMEs through the heliosphere, and impacts on Solar System bodies. In addition, we identify promising studies of the last phase of CME activity and of global imaging of ejecta in the heliosphere. It is clear that this area will be extremely productive in the coming months and years.

\section{Comments on CMEs, EIT-waves and flare/CME symmetry}

EIT or coronal waves have become a hot topic. These are rapidly expanding disturbances which propagate around the solar globe, and they are shown to have a close association with CMEs and flares. Biesecker et al. (2002) studied 173 EIT-waves and showed an intimate association with CMEs and a less significant flare association. Of interest here is the CME association because it may provide some insight to CME activity. Amongst the models being proposed for this phenomenon, Plunkett et al. (2002) suggested that the waves are due to fast-mode MHD waves propagating from CME initiation sites. More recently, Attrill et al. (2007) have suggested that the 'waves' are actually due to successive reconnections in the flanks of a CME.

We must address two misconceptions. First, the apparent dimming displayed in differenced EUV images behind the expanding EIT-wave is not the same as the so-called coronal dimming phenomenon. Such coronal dimming is more localised, does not display such rapid expansion, and reveals a much more significant decrease in intensity, though such an event may be encompassed within the area behind an EIT-wave. However, Chen and Fang (2005), for example, suggest that EIT-wave defines a coronal dimming region and refers to the dimming work of Harrison et al. (2003). That work was actually concerned with coronal dimming of a large but localised region under a CME and not the shallow dimming found over a large area of the solar globe behind an EIT-wave.

A second, and perhaps more significant, misconception is the association between the legs of the CME and the EIT-wave-front. For example, the Chen and Fang (2005) paper mentioned above discussed a model in which the CME extent (and the coronal dimming) was defined explicitly by the extent of the EIT-wave, i.e. the EIT-wave defined the lateral extent of the ascending CME footprint (and the dimming event). Attrill et al. (2007) also state that the coronal wave is the magnetic footprint of a CME; specifically they state that "the diffuse EIT coronal bright fronts are due to driven magnetic reconnections between the skirt of the expanding CME magnetic field and favourably oriented quiet Sun magnetic loops". The EIT-wave may well be due to an MHD wave propagating through the corona, or to magnetic reconnection processes, as suggested by Attrill et al. (2007). However, we know from decades of observation that CME legs do not separate significantly with time and certainly do not expand to wrap around the solar disk. Thus, it is imperative that we do not associate intimately the expanding EIT "wave" with the 
legs of a CME. That is not to say that they cannot be associated at all - they are just not co-located throughout the sequence of events.

Nobody doubts the close association between flares and CMEs. From an analysis of a number of flare events associated with CMEs detected using the Solar Maximum Mission coronagraph, Harrison $(1986,1995)$ demonstrated asymmetry between flare and associated CME activity. They suggested that a flare associated with a CME could lie anywhere under the CME-span. At the same time, they claimed that the flare and CME onset times were not always coincident - the onset of the CME could appear to precede the associated flare onset. In addition, they pointed out that the CME-spans were an order of magnitude (or more) larger than the scale of associated flares and, in the absence of clear expansion of CME-legs, suggested that CME source regions must be much larger than the flare site. All of this led to the suggestion that the flare and CME are closely associated but that they do not cause one another - they both result from the relaxation of complex magnetic topologies and, as such, can occur in concert (see Harrison 1991).

Many subsequent papers have provided evidence to confirm or deny this scenario. Yashiro et al. (2008) examined 496 flare-CME pairs using SOHO data in an essential study, extending the old analyses significantly. Their principal conclusions were:

1. $\mathrm{X}$ and $\mathrm{M}$ class flares associated with CME onsets are likely to show symmetry within the CME-span, i.e. to lie under the centre of the CME-span;

2. C-class flares showed less symmetry with significant numbers residing near the edge or outside the CME-span.

Yashiro et al.'s Figure 4 uses a flare-CME symmetry index used in previous studies and claim that the data show consistency with the so-called CSHKP flare-CME model. This is a combination of models due to Carmichael, Sturrock, Hirayama, Kopp and Pneumann, which calls for reconnection above the flare site with the ascending CME propagating symmetrically above the flare. For this model there is clear symmetry between the CME and associated flare and the onsets of the flare and CME should coincide. Whilst the symmetry displayed by many of the $\mathrm{X}$ and $\mathrm{M}$ class flare events appears to be consistent with this approach, their results do present an anomaly for several reasons:

1. Although the $\mathrm{M}$ and $\mathrm{X}$ class events show a high degree of symmetry between flare and $\mathrm{CME}$, there is a tail to the distribution which shows that some $\mathrm{M}$ and $\mathrm{X}$ class flares occur anywhere under the CME-span.

2. The Yashiro et al. (2008) data for the C-class events actually shows the same result as the Harrison et al. papers, the flares appear to occur anywhere under the CME-span.

3. We also note that there are flares without obvious CME activity.

The Harrison et al. studies were taken from a period lacking in bright flares, so the results of Yashiro et al. (2008) are not inconsistent with their results. However, if we wish to adopt the CSHKP approach for the brighter flares, we cannot use that approach for the flares indicated in the three points above. Do we adopt a different flare-CME model for weaker flares and for some of the large flares, not to mention the flare-less CMEs? The author is uncomfortable with the notion that we should devise a flare model that only supports the brighter flares which showed symmetry. We require a model which allows for any symmetry/asymmetry between flare and CME and in the opinion of the author that means adopting the non-cause and effect approach mentioned above.

\section{Conclusions}

There are key open issues that require clarification or general acceptance, to progress in some areas of CME research. We have addressed coronal dimming and stressed that recent spectroscopic interpretations have far-reaching consequences and must be taken 
seriously. We have briefly reviewed the opening field of heliospheric imaging; it is clear that we have witnessed rapid progress and there is promise of much to come. There are also some issues relating to EIT-waves and our understanding of how they are associated with CME footprints, and to the flare-CME asymmetry that need careful consideration.

\section{References}

Attrill G. D. R., Harra, L. K., van Driel-Gesztelyi, L., \& Demoulin, P. 2007, ApJ 656, L101

Bewsher, D., Harrison, R. A., \& Brown, D. S. 2008, A\& A 478, 897

Crooker, N. U. \& Horbury, T. S. 2006, Space Sci. Revs 123, 93

Biesecker D. A., Myers, D. C., Thompson, B. J., Hammer, D. M., \& Vourlidas, A., 2002, Astrophys. J., 569, 1009

Chen, P. F. \& Fang, C. 2005 in 'Coronal and Stellar Mass Ejections', Proc. IAU Symp. 226, 55

Davies J. A., Harrison, R. A., Rouillard, A. P., Sheeley, N. R., Perry, C. H., Bewsher, D., Davis, C. J., Eyles, C. J., Crothers, S. R., \& Brown, D. S. 2009, Geophys. Res. Lett. 36, L02102

Eyles C. J., Simnett, G. M., Cooke, M. P., Jackson, B. V., Buffington, A., Hick, P. P., Waltham, N. R., King, J. M., Anderson, P. A., Holladay, P. E. 2003 Solar Phys. 217, 319

Eyles C. J., Harrison, R. A., Davies, C. J., Waltham, N. R., Shaughnessy, B. M., MapsonMenard, H.C. A., Bewsher, D., Crothers, S. R., \& Davies, J. A. 2009, Solar Phys. in press

Gopalswamy N. and Hanaoka, Y. 1998, ApJ 498, 179

Gosling, J. T., Birn, J., Hesse, M. 1995, Geophys. Res. Lett. 22, 869

Harra, L. K. \& Sterling, A. C. 2001, ApJ 561, L215

Harra, L. K., Hara, H., Imada, S., Young, P. R., Williams, D., Sterling, A., Korendyke, C., \& Attrill, G. 2007, Publ. Astron. Soc. Japan 59, S801

Harrison, R. A. 1986, A\& A 162, 283-291

Harrison R. A. 1991, Phil. Trans. Roy. Soc. London, A, 336, 401-412

Harrison R. A. 1995, A\&A 304, 585-594

Harrison R. A. 2006, in 'Solar eruptions $\&$ energetic particles', AGU Geophys. Mon. Ser. 165, 73

Harrison R. A. \& Bewsher, D. 2007, A\&A 461, 1155

Harrison R. A. \& Lyons, M. 2000, A\&A 358, 1097

Harrison R. A., Bryans, P., Simnett, G. M., \& Lyons, M. 2003, A $\mathscr{S} A$ 400, 1071

Harrison R. A., Davis, C. J., Eyles, C. J., \& 12 co-authors 2008, Solar Phys. 247, 171

Harrison, R. A., Davies, J. A., Rouillard, A. P., Davis, C. J., Eyles, C. J., Bewsher, D., Crothers, S. R., Howard, R. A., Sheeley, N. R., Vourlidas, A., Webb, D. F., Brown, D. S., \& Dorrian, G. D. 2009a, Solar Phys. in press

Harrison, R. A., Davis, C. J., Bewsher, D., Davies, J. A., Eyles, C. J., \& Crothers, S. R. 2009b, Adv. Space Res. submitted

McComas D. J. 1995, Rev. Geophys. Suppl. 33, 603

Plunkett, S. P., Michels, D. J., Howard, R. A., \& 6 co-authors, 2002, Adv. Sp. Res. 29, 1473

Rouillard, A., Davies, J. A., Forsyth, R., J., \& 9 co-authors, 2008a, Geophys. Res. Lett. 35, L10110

Rouillard, A. P., Davies, J. A., Rees, A., \& 13 co-authors, 2008b, J. Geophys. Res. submitted Rust, D. M. \& Hildner, E. 1976, Solar Phys. 48, 381

Sheeley, N. R., Walters, J. H., Wang, Y.-M. \& Howard, R. A. 1999, J. Geophys. Res. 104, 24,739

Sheeley, N. R., Herbst, A. D., Palatchi, C. A., \& 21 co-authors, 2008a, ApJ 674, L109

Sheeley, N. R., Herbst, A. D., Palatchi, C. A., \& 21 co-authors, 2008b, ApJ 675, 853

Sterling, A. C. \& Hudson, H. S. 1997, ApJ 491, L55

Vourlidas, A. \& Howard, R. A. 2006, ApJ 642, 1216

Vourlidas, A., Davis, C. J., Eyles, C. J., Crothers, S. R., Harrison, R. A., Howard, R. A., Moses, J. D., \& Socker, D. G. 2007, ApJ 668, L79

Yashiro, S., Michalek, G., Akiyama, S., Gopalswamy, N., \& Howard, R. A. 2008b, ApJ 673, 1174

Zarro, D. M., Sterling, A. C., Thompson, B. J., Hudson, H. S., \& Nitta, N. 1999, ApJ 520, 139 